\title{
MATHEMATIKA
}

A JOURNAL OF PURE AND APPLIED MATHEMATICS

$\begin{array}{llll}\text { Vol. } 27 \text { Part } 2 . & \text { December } 1980 & \text { No. } 54 .\end{array}$

\section{ON A THEOREM OF BOMBIERI-VINOGRADOV TYPE}

\author{
E. FOUVRY AND H. IWANIEC +
}

§1. Introduction. The celebrated theorem of Bombieri and A. I. Vinogradov states that

$$
\sum_{q<x^{(1 / 2)-\varepsilon}} \max _{(a, q)=1}\left|\pi(x ; q, a)-\frac{\pi(x)}{\phi(q)}\right| \ll x(\log x)^{-A},
$$

for any $\varepsilon>0$ and $A>0$, the implied constant in the symbol $\ll$ depending at most on $\varepsilon$ and $A$ (see [1] and [14]). The original proofs of Bombieri and Vinogradov were greatly simplified by P. X. Gallagher [4]. An elegant proof has been given recently by $R$. C. Vaughan [13]. For other references see H. L. Montgomery [10] and H. -E. Richert [12]. Estimates of type (1) are required in various applications of sieve methods. Having this in mind distinct generalizations have been investigated (see for example [15] and [2]). Y. Motohashi established a general theorem which, roughly speaking, says that if (1) holds for two arithmetic functions then it also holds for their Dirichlet convolution; for precise assumptions and statement see [11]. So far, all methods depend on the large sieve inequality (see [10])

$$
\sum_{q \leqslant Q} \sum_{\chi(\bmod q)}^{*}\left|\sum_{n \leqslant N} a_{n} \chi(n)\right|^{2} \ll\left(Q^{2}+N\right) \sum_{n \leqslant N}\left|a_{n}\right|^{2},
$$

which sets the limit $x^{1 / 2}$ for the modulus $q$ in (1) and in its generalizations.

It is the aim of this paper to present arguments which yield theorems of Bombieri-Vinogradov type with an extended range for $q$. We shall treat carefully

$$
\pi(x, z ; q, a)=\sum_{\substack{n \leq x \\ n \equiv a(\bmod q)}} f_{z}(n)
$$

where $f_{z}$ is the characteristic function of the set of integers $n$ having no prime factor less than $z$. Let us introduce also

$$
\pi(x ; z, q)=\sum_{\substack{n \leq x \\(n, q)=1}} f_{z}(n) .
$$


We have proved the following

Theorem. Let $z \leqslant x^{1 / 883}$ and $1 \leqslant|a| \leqslant x$. Then, for any $A>0$,

$$
\sum_{\substack{q<x^{11 / 21} \\(q, a)=1}}\left|\pi(x, z ; q, a)-\frac{1}{\phi(q)} \pi(x, z ; q)\right| \ll x(\log x)^{-A},
$$

the implied constant depending only on $A$.

Our method applies to a wide class of arithmetic functions $f(n)$, for which the sum

$$
\pi_{f}(x ; q, a)=\sum_{\substack{n \leq x \\ n \equiv a(\bmod q)}} f(n)
$$

can be rearranged as a sum

$$
\sum_{\substack{L<2 L \\ l m=a(\bmod q)}} \sum_{\substack{M<m \\ l m}} \alpha_{l} \beta_{m}
$$

of bilinear forms, with the variables of summation $l$ and $m$ in appropriate intervals. Such a representation for $f_{z}(n)$ is obtained through a combinatorial sieve identity (see Lemma 1). We failed to obtain (3) in the most interesting case $z=x^{1 / 2}$, in other words for $f(n)=\Lambda(n)$. In the latter case, Vaughan's identity (see [13]) would serve as a bilinear form (4), but unfortunately with $L$ and $M$ not well enough controlled for our method to apply.

Acknowledgement. The authors express their gratitude to Professor $\mathrm{H}$. Halberstam for pointing out some errors and for some helpful remarks about the first version of this paper. Our work was done when the second author enjoyed a one year visit to the University of Bordeaux I. It is a great pleasure for him to take this opportunity to speak of the pleasing scientific atmosphere in which collaboration was so fruitful.

§2. Sketch of the main ideas. The bilinear form (4) is approximated by

$$
\frac{1}{\phi(q)} \sum_{L<l \leqslant 2 L} \sum_{\substack{M<m \leqslant 2 M \\(m, q)=1}} \alpha_{l} \beta_{m}
$$

with the total error less than

$$
R(M, L ; Q)=\sum_{\substack{Q<q \leqslant 2 Q \\(q, a)=1}} \sum_{\substack{M<m \leqslant 2 M \\(m, q)=1}}\left|\sum_{\substack{L<l \leqslant 2 L \\ l m \equiv a(\bmod q)}} \alpha_{l}-\frac{1}{\phi(q)} \sum_{\substack{L<l \leqslant 2 L \\(l, q)=1}} \alpha_{l}\right|
$$


The problem of bounding $R(M, L ; Q)$ is reduced, by the Cauchy-Schwarz inequality

$$
R(M, L ; Q) \leqslant(Q M)^{1 / 2} D^{1 / 2}(M, L ; Q),
$$

to that of bounding the dispersion

$$
\begin{aligned}
D(M, L ; Q) & =\sum_{q} \sum_{m}\left(\sum_{\substack{L<l \leqslant 2 L \\
l m=a(\bmod q)}} \alpha_{l}-\frac{1}{\phi(q)} \sum_{\substack{L<l \leqslant 2 L \\
(l, q)=1}} \alpha_{l}\right)^{2} \\
& =W(M, L ; Q)-2 V(M, L ; Q)+U(M, L ; Q),
\end{aligned}
$$

say. Each of the terms $U, V$ and $W$ is evaluated separately, the most difficult being $W$. By definition

$$
W(M, L ; Q)=\sum_{\substack{Q<4 \leqslant 2 Q \\(q, a)=1}} \sum_{\substack{M<m \leqslant 2 M \\(m, q) \leq 1}} \sum_{\substack{L<l_{1}, l_{2} \leqslant 2 L \\ l, m=l_{2} m \equiv a(\bmod q)}} \alpha_{l_{1}} \alpha_{l_{2}} .
$$

With an admissible error we may replace $W(M, L ; Q)$ by $W^{*}(M, L ; Q)$, which stands for the same sum with the range of summation restricted to $\left(l_{1}, l_{2}\right)=1$, $l_{1} \equiv l_{2}(\bmod q)$. In particular the diagonal terms $l_{1}=l_{2}$ disappear.

When treating $W^{*}(M, L ; Q)$ we carry out the summation over $m$ first. We use $\bar{l}_{1}$ to denote the reciprocal of $l_{1}$ modulo $q$, so that $l_{1} \bar{l}_{1} \equiv 1 \bmod q$. Writing

$$
\sum_{\substack{M<m \leqslant 2 M \\ m=a I_{1}(\bmod q)}} 1=\frac{M}{q}+r\left(q, a \bar{l}_{1}\right)
$$

it is trivial that $\left|r\left(q, a \bar{l}_{1}\right)\right| \leqslant 1$, but this turns out to be not satisfactory. We obtain a great cancellation of the errors $r\left(q, a \bar{l}_{1}\right)$ in the sums over $l_{1}, l_{2}$ and $q$. By expanding each $r\left(q, a I_{1}\right)$ into a Fourier series, a typical term to be considered is

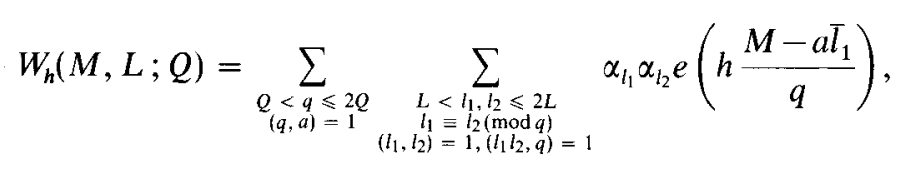

with $h \neq 0$. Since $Q$ will be nearly as large as $L$ and $l_{1} \equiv l_{2}(\bmod q)$ there is not much room for summation over $l_{1}$ and $l_{2}$. For this reason we reinterpret the condition $l_{1} \equiv l_{2}(\bmod q)$ by writing

$$
l_{1}-l_{2}=q r \quad \text { with } 0<|r| \leqslant L / Q,\left(r, l_{1} l_{2}\right)=1 .
$$

Here $r$ is rather small, so the condition $l_{1} \equiv l_{2}(\bmod r)$ constrains the variables $l_{1}, l_{2}$ less than does $l_{1} \equiv l_{2}(\bmod q)$. In addition,

$$
\frac{M-a \bar{l}_{1}}{q} \equiv\left(M-\frac{a}{l_{1}}\right) \frac{r}{l_{1}-l_{2}}-\operatorname{ar} \frac{\bar{l}_{2}}{l_{1}}(\bmod 1) .
$$


Therefore we arrive at sums of the type

$$
\sum_{0<|r|<L / Q} \sum_{\substack{L<l_{1}, l_{2} \leqslant L_{1} \\\left(l_{1}, l_{2}\right)=1,\left(l_{1}-l_{2}, a r\right)=r}} \alpha_{l_{1}} \alpha_{l_{2}} e\left(-a h r \frac{I_{2}}{l_{1}}\right),
$$

with some $L_{1} \in(L, 2 L]$, the factor

$$
e\left(\left(M-\frac{a}{l_{1}}\right) \frac{h r}{l_{1}-l_{2}}\right)
$$

being removed by partial summation. A connection with the incomplete Kloosterman sums is suggested. By the Cauchy-Schwarz inequality,

$$
\left|\sum_{l_{1}, l_{2}} \alpha_{l_{1}} \alpha_{l_{2}} e\left(-a h r \frac{\bar{l}_{2}}{l_{1}}\right)\right|^{2} \leqslant L \sum_{l^{\prime}, l^{\prime \prime}} \alpha_{l^{\prime}} \bar{\alpha}_{l^{\prime \prime}} \sum_{l} e\left(-a h r \frac{\left(l^{\prime}-l^{\prime \prime}\right) \bar{l}}{l^{\prime} l^{\prime \prime}}\right) .
$$

Using Weil's estimate of $\sum_{l}$ one just fails to get a non-trivial bound because the modulus $l^{\prime} l^{\prime \prime}$ is as large as the square of the length of the incomplete Kloosterman sum $\sum_{l}$. Hooley's conjecture $R^{*}$ (see [7]) would be helpful. In order to avoid any unproved hypothesis, we appeal to a particular property of the coefficients $\alpha_{l}$ to rearrange the sum $\sum_{l_{1}, l_{2}}$ into another bilinear form with variables of summation of a different order of magnitude. Then, the above procedure yields incomplete Kloosterman sums which are manageable by Weil's estimate. We doubt whether the elementary result of Kloosterman [9] is sufficient.

From the main terms in the dispersion $D(M, L ; Q)$ we get

$$
M \sum_{\substack{Q<q \leqslant 2 Q \\(q, q)=1}} \phi^{-2}(q) \sum_{\substack{\lambda(\bmod q) \\(\lambda, q)=1}}\left|\sum_{\substack{L<l \leqslant 2 L \\ l=\lambda(\bmod q)}} \alpha_{l}-\frac{1}{\phi(q)} \sum_{\substack{c<l \leqslant 2 L \\(l, q)=1}} \alpha_{l}\right|^{2} .
$$

We estimate this by applying the large sieve inequality and the Siegel-Walfisz theorem in a way familiar from the Barban and Davenport-Halberstam theorem.

§3. Lemmas. Let $P(z)=\prod_{p<z} p$ for $z \geqslant 2$. Let $F(n)$ be an arithmetic function vanishing for almost all $n$. By the Buchstab identity

$$
\sum_{n} f_{z}(n) F(n)=\sum_{n} F(n)-\sum_{p<z}\left(\sum_{n \equiv 0(\bmod p)} f_{p}(n) F(n)\right)
$$

on applying the 'exclusion-inclusion principle' familiar from combinatorial sieve theory (see [5] and [8]) we obtain

Lemma 1. Let $D \geqslant z \geqslant 2$. Then

$$
\sum_{n} f_{z}(n) F(n)=\sum_{d \mid P(z)} \lambda_{d}\left(\sum_{n \equiv 0\langle\bmod d)} F(n)\right)+\sum_{d \mid P(z)} \sigma_{d}\left(\sum_{n \equiv 0(\bmod d)} f_{p(d)}(n) F(n)\right),
$$


where $p(d)$ stands for the least prime factor of $d$, and, for a square-free $d=p_{1} \ldots p_{r}>1, p_{1}>\ldots>p_{r}$, we define

$$
\lambda_{d}= \begin{cases}(-1)^{r}, & \text { if } p_{1} \ldots p_{t} p_{l}<D \text { for all } l \leqslant r \\ 0, & \text { otherwise }\end{cases}
$$

and

$$
\sigma_{d}= \begin{cases}(-1)^{r}, & \text { if } p_{1} \ldots p_{l} p_{l}<D \text { for all } l<r \text { and } p_{1} \ldots p_{r} p_{r} \geqslant D \\ 0, & \text { otherwise. }\end{cases}
$$

For $d=1$ we define $\lambda_{1}=1$ and $\sigma_{1}=0$.

Note that if $\lambda_{d} \neq 0$ then $d<D$, and if $\sigma_{d} \neq 0$ then $D / \tilde{p}(d) \leqslant d<D$. Hence we obtain the

Corollary. Let $D \geqslant z \geqslant 2$. Then

$$
\left|\sum_{n} f_{z}(n) F(n)\right| \leqslant \sum_{\substack{d<D \\ d \mid P(z)}}\left|\sum_{l} F(d l)\right|+\sum_{p<z \quad D_{D / p^{2} \leqslant d<D / p} \sum_{p d \mid P(z)}}\left|\sum_{l} f_{p}(l) F(d l p)\right| .
$$

The following result is known in sieve theory as a 'fundamental lemma' (see [5]).

Lemma 2. For $R, z \geqslant 2$ and $(\alpha, q)=1$ we have

$$
\sum_{\substack{n \leqslant x \\ n \equiv \alpha(\bmod q)}} f_{z}(n)=\frac{x}{q} \prod_{\substack{p<z \\ p \nmid q}}\left(1-\frac{1}{p}\right)\left\{1+O\left(e^{-s}\right)\right\}+O(R),
$$

where $s=\log R / \log z$. The implied constants are absolute.

Lemma 3. If $\tau(q)$ is the number of divisors of $q$,

$$
\sum_{\substack{n \leqslant x \\(n, q)=1}} 1=\frac{\phi(q)}{q} x+O(\tau(q)) .
$$

Lemma 4. Let $\psi(\xi)=\xi-[\xi]-\frac{1}{2}$ and $\Delta>0$. There are two functions $A(\xi)$ and $B(\xi)$ periodic in $\xi(\bmod 1)$ such that

with

$$
\begin{aligned}
& |\psi(\xi)-A(\xi)| \leqslant B(\xi) \\
& A(\xi)=\sum_{h \neq 0} \hat{A}(h) e(h \xi) \\
& B(\xi)=\sum_{h \neq 0} \hat{B}(h) e(h \xi)+\Delta
\end{aligned}
$$

$$
\hat{A}(h), \hat{B}(h) \ll C_{h}=\min \left(\frac{1}{|h|}, \frac{1}{\Delta^{3} h^{4}}\right),
$$


and $e(z)=e^{2 \pi i z}$.

Proof., Take functions $A(\xi)$ and $B(\xi)$ of class $C^{3}$ whose graphs are

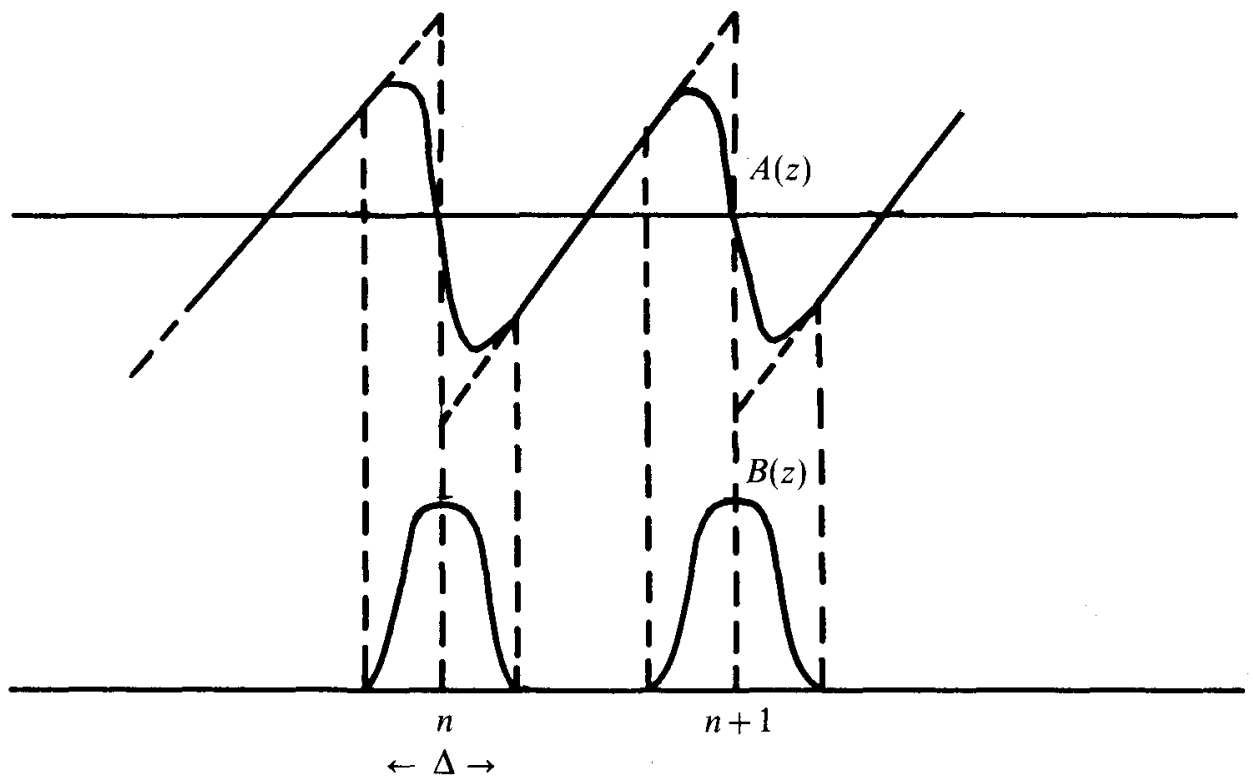

and whose derivatives satisfy $A^{(p)}(\xi), B^{(p)}(\xi) \ll \Delta^{-p}$ for $p \leqslant 3$, (compare with Lemma 2 of [3]).

The next lemma is a consequence of Weil's estimate for Kloosterman's sums. The proof is similar to Lemma 3 of Hooley [6].

Lemma 5. Let $0<A_{2}-A_{1} \leqslant b$. Then

$$
\sum_{\substack{A_{1}<a \leqslant A_{2} \\(a, b c)=1 \\ a \equiv \lambda(\bmod \Lambda)}} e\left(d \frac{\bar{a}}{b}\right) \ll(b, d)^{1 / 2} b^{1 / 2} \tau(b c) \log 2 b .
$$

The implied constant is absolute. The notation $\bar{a}$ used when writing $\bar{a} / b$ or in $a$ congruence $(\bmod b)$ means that $a \bar{a} \equiv 1(\bmod b)$.

LEMMA 6. For any pair $a, b$ of non-zero coprime integers,

$$
\frac{\bar{a}}{b}+\frac{\bar{b}}{a} \equiv \frac{1}{a b}(\bmod 1)
$$

Lemma 7. If $\chi$ is a non-principal character $\bmod d$, and $d \leqslant(\log \xi)^{A}$, then

$$
\sum_{n \leqslant \xi} \chi(n) f_{z}(n) \ll \xi \exp \left(-(\log \xi)^{1 / 5}\right)
$$

for any $A>0$, the implied constant depending only on $A$. 
Proof. By Buchstab's identity,

$$
\begin{aligned}
\sum_{n \leqslant \xi} \chi(n) f_{z}(n) & =\sum_{n \leqslant \xi} \chi(n)-\sum_{p<z} \chi(p) \sum_{n \leqslant \xi / p} \chi(n) f_{p}(n) \\
& \ll d+\sum_{p<z_{1}}\left|\sum_{n \leqslant \xi / p} \chi(n) f_{p}(n)\right|+\left|\sum_{z_{1} \leqslant p<z} \chi(p) \sum_{n \leqslant \xi / p} \chi(n) f_{p}(n)\right|,
\end{aligned}
$$

where $z_{1}=\min (z, \exp \sqrt{\log \xi})$. Letting $R=\xi^{1 / 2}$ in the 'fundamental lemma' we obtain

$$
\begin{aligned}
\sum_{p<z_{1}}\left|\sum_{n \leqslant \xi / p} \chi(n) f_{p}(n)\right| & =\sum_{p<z_{1}} \mid \sum_{l(\bmod d)} \chi(l)\left[\frac{\xi}{p d} \prod_{\substack{\left.p_{1}<p \\
p_{1}\right\} d}}\left(1-\frac{1}{p_{1}}\right)\left\{1+O\left(e^{-\varsigma p}\right)\right\}+O(R)\right] \\
& \ll \xi \exp \left(-\frac{\log R}{\log z_{1}}\right)+R d z_{1} \ll \xi \exp \left(-\frac{1}{2}(\log \xi)^{1 / 2}\right),
\end{aligned}
$$

where $s_{p}=\log R / \log p \geqslant \log R / \log z_{1} \geqslant \frac{1}{2}(\log \xi)^{1 / 2}$. The second double sum is empty if $z \leqslant \exp (\sqrt{\log \xi})$; thus we assume that $z>\exp (\sqrt{\log \xi})=z_{1}$, and we obtain

$$
\begin{aligned}
\sum_{z_{1} \leqslant p<z} \chi(p) \sum_{n \leqslant \xi / p} \chi(n) f_{p}(n) & =\sum_{n \leqslant \xi / z 1} \chi(n) \sum_{z_{1}<p \leqslant \min (z, \xi / n, p(n))} \chi(p) \\
& \ll \sum_{n \leqslant \xi / z 1} \frac{\xi}{n} \exp \left(-c \sqrt{\log z_{1}}\right) \ll \xi \exp \left(-(\log \xi)^{1 / 5}\right),
\end{aligned}
$$

by the Siegel-Walfisz theorem. This completes the proof.

COROLlary. Under the same assumptions,

$$
\sum_{\substack{n<\zeta \\(n, e)=1}} \chi(n) f_{z}(n) \ll \tau(e) \xi \exp \left(-(\log \xi)^{1 / 6}\right)
$$

\$4. Reduction of the problem. We split up the sum $(3)$ into $\ll(\log x)^{2}$ sums of type

$$
S(y, Q)=\sum_{\substack{Q<q \leqslant 2 Q \\(q, a)=1}}\left|\sum_{\substack{y<n \leqslant 2 y \\ n=a(\bmod q)}} f_{z}(n)-\frac{1}{\phi(q)} \sum_{\substack{y<n \leqslant 2 y \\(n, q)=1}} f_{z}(n)\right|,
$$

with $2 y \leqslant x$ and $2 Q \leqslant x^{1 / 21}$. It is sufficient to show that

$$
S(y, Q) \ll x(\log x)^{-A-2},
$$

for $z \leqslant x^{1 / 883}$ and $1 \leqslant|a| \leqslant x$. We have trivially that

$$
S(y, Q) \ll \sum_{Q<q \leqslant 2 Q}\left(\frac{y}{\phi(q)}+1\right) \ll y+Q,
$$


so that (5) is obvious for $y \leqslant x(\log x)^{-A-2}$. In what follows we assume that

$$
x(\log x)^{-A-2}<y \leqslant x .
$$

Now we want to rearrange $S(y, Q)$ as a sum of bilinear forms. For this, apply Lemma 1 twice to the characteristic function of the set of integers $n \in(y, 2 y], n \equiv a(\bmod q)$ and to the characteristic function of the set of integers $n \in(y, 2 y],(n, q)=1$. Then subtract $1 / \phi(q)$ times the second inequality from the first, to obtain, as in the corollary to Lemma 1 ,

$$
\begin{aligned}
& \left|\sum_{\substack{y<n \leqslant 2 y \\
n \equiv a(\bmod q)}} f_{z}(n)-\frac{1}{\phi(q)} \sum_{\substack{y<n \leqslant 2 y \\
(n, q)=1}} f_{z}(n)\right| \\
& \leqslant \sum_{\substack{d<D \\
d \mid P(z) \\
(d, q)=1}}\left|\sum_{\substack{y<n \leqslant 2 y \\
n \equiv a(\bmod q) \\
n \equiv 0(\bmod d)}} 1-\frac{1}{\phi(q)} \sum_{\substack{y<n \leqslant 2 y \\
n \equiv 0(\bmod d) \\
(n, q)=1}} 1\right| \\
& +\sum_{p<z} \sum_{\substack{1 \\
D p^{-2} \leq d<D_{p}-1 \\
p d \mid P(z) \\
(p d, q)=1}}\left|\sum_{\substack{y<n \leqslant 2 y \\
n \equiv a(\bmod q) \\
n=0(\bmod d p)}} f_{p}(n)-\frac{1}{\phi(q)} \sum_{\substack{y<n \leqslant 2 y \\
n \equiv 0(\bmod p d) \\
(n, q)=1}} f_{p}(n)\right| \\
& =S_{1}(q, D)+\sum_{\substack{p<z \\
p \nmid q}} S_{p}(q, D),
\end{aligned}
$$

say. Hence, in the above notation

$$
\begin{aligned}
|S(y, Q)| & \leqslant \sum_{\substack{Q<q \leqslant 2 Q \\
(q, a)=1}} S_{1}(q, D)+\sum_{\rho<z} \sum_{\substack{Q<q \leqslant 2 Q \\
(q, a p)=1}} S_{p}(q, D) \\
& =S_{1}(y, D, Q)+\sum_{p<z} S_{p}(y, D, Q),
\end{aligned}
$$

say. The sums $S_{p}(y, D, Q)$ with $p<\min (z, \exp (\sqrt{\log x}))=z_{0}$, say, will be estimated easily by means of Lemmas 2 and 3 while those with larger $p$ will be treated by a dispersion method.

§5. Estimate of $S_{1}(y, D, Q)$. For $(d, q)=1$ we have

$$
\sum_{\substack{y<n \leqslant 2 y \\ n \equiv a(\bmod q) \\ n \equiv 0(\bmod d)}} 1=\frac{y}{q d}+O(1),
$$

and, by Lemma 3 ,

$$
\sum_{\substack{y<n \leqslant 2 y \\ n=0(\bmod d) \\(n, q)=1}} 1=\frac{\phi(q)}{q} \frac{y}{d}+O(\tau(q)) .
$$


Hence $S_{1}(q, D) \ll D$ and consequently

$$
S_{1}(y, D, Q) \ll Q D \ll x^{1-2 \varepsilon},
$$

provided

$$
Q D \ll x^{1-2 \varepsilon},
$$

which we henceforth assume.

§6. Estimate of $\sum_{p<z_{0}} S_{p}(y, D, Q)$. By Lemma 2, for each $\alpha$ with $(\alpha, q)=1$, we have

$$
\sum_{\substack{y<p m n \leqslant 2 y \\ p m n \equiv x(\bmod q)}} f_{p}(n)=\frac{y}{p m q} \prod_{\substack{p_{1}<p \\ p_{1} \nmid q}}\left(1-\frac{1}{p_{1}}\right)\left\{1+O\left(e^{-S_{p}}\right)\right\}+O(R),
$$

where $R$ is any number $\geqslant p$ and $s_{p}=\log R / \log p$. Hence

$$
S_{p}(q, D) \ll \sum_{D p^{-2} \leqslant m \leqslant D p^{-1}}\left(\frac{y}{p q m} e^{-S_{p}}+R\right) \ll \frac{y}{q} \frac{\log p}{p} e^{-S_{p}}+\frac{D R}{p}
$$

and consequently

$$
S_{p}(y, D, Q) \ll y \frac{\log p}{p} e^{-S_{p}}+\frac{D Q R}{p} .
$$

For $R=x^{\varepsilon / 2}$ this bound yields

$$
\sum_{p<z_{0}} S_{p}(y, D, Q) \ll x \exp \left(-(\log x)^{1 / 3}\right)
$$

the implied constant depending on $\varepsilon$ only.

Now we proceed to estimate $S_{p}(y, D, Q)$ with $z_{0} \leqslant p<z$.

\$7. Rearrangement of $S_{p}(y, D, Q)$. Let $M$ take the values $2^{-\lambda} D p^{-1}$ for $\lambda=1,2, \ldots$ such that $D p^{-2} \leqslant M<D p^{-1}$, so that there are at most $2 \log p$ such $M$ 's. We split up $S_{p}(y, D, Q)$ into $\ll \log p$ sums of the type

$$
E_{p}(y, M, Q)=\sum_{\substack{Q<q \leqslant 2 Q \\(q, a p)=1}} \sum_{\substack{M<m \leqslant 2 M \\(m, q)=1}}\left|\sum_{\substack{y<p m n \leqslant 2 y \\ p m n \\=a(p \bmod q) \\(p, n)=1}} f_{p}(n)-\frac{1}{\phi(q)} \sum_{\substack{y<p m n \leqslant 2 y \\(n, p q)=1}} f_{p}(n)\right|,
$$

thus obtaining

$$
S_{p}(y, D, Q) \leqslant \sum_{M} E_{p}(y, M, Q)+O\left(y p^{-2} \log x\right) .
$$


Here, the error term comes from the contribution of $n$ 's divisible by $p^{2}$. This error is admissible because

$$
\sum_{z 0 \leqslant p<z} y p^{-2} \log x \ll y(\log x) \exp (-\sqrt{\log x}) .
$$

§8. The dispersion. By the Cauchy-Schwarz inequality we obtain

$$
E_{p}^{2}(y, M, Q) \leqslant M Q D_{p}(M, Q)
$$

where $D_{p}(M, Q)$, called the dispersion, stands for

$$
\begin{aligned}
D_{p}(M, Q) & =\sum_{\substack{Q<q \leqslant 2 Q \\
(q, a p)=1}} \sum_{\substack{M<m \leqslant 2 M \\
(m, q)=1}}\left(\sum_{\substack{y<p m n \leqslant 2 y \\
p m n \\
=(n) \bmod q)}} f_{p}(n)-\frac{1}{\phi(q)} \sum_{\substack{y<p m n \leqslant 2 y \\
(n, p q)=1}} f_{p}(n)\right)^{2} \\
& =W_{p}(M, Q)-2 V_{p}(M, Q)+U_{p}(M, Q),
\end{aligned}
$$

say. Each term $U_{p}, V_{p}$ and $W_{p}$ will be evaluated separately. By definition,

$$
U_{p}(M, Q)=\sum_{\substack{Q<q \leqslant 2 Q \\(q, a p)=1}} \sum_{\substack{M<m \leqslant 2 M \\(m, q)=1}}\left(\frac{1}{\phi(q)} \sum_{\substack{<<p m n \leqslant 2 y \\(n, p q)=1}} f_{p}(n)\right)^{2}
$$

§. Evaluation of $V_{p}(M, Q)$. By definition,

$$
V_{p}(M, Q)=\sum_{\substack{Q<q \leqslant 2 Q \\(q, a p)=1}} \frac{1}{\phi(q)} \sum_{\substack{N / 2<n_{1}, n_{2}<2 N \\\left(n_{1} n_{2}, p q\right)=1 \\ 1 / 2<n_{1} / n_{2}<2}} f_{p}\left(n_{1}\right) f_{p}\left(n_{2}\right) \sum_{\substack{M_{1}<m \leqslant M_{2} \\ m \equiv a \overline{n_{1}}(\bmod q)}} 1,
$$

where for simplicity we have written $N=y / p M, M_{1}=\max \left(M, y /\left(p n_{1}\right), y /\left(p n_{2}\right)\right)$ and $M_{2}=\min \left(2 M, 2 y /\left(p n_{1}\right), 2 y /\left(p n_{2}\right)\right)$. We carry out the summation over $m$ first. Trivially we would take $\left(M_{2}-M_{1}\right) q^{-1}+O(1)$ but this is useless because $M$ is going to be smaller than $Q$. Therefore we are looking for an explicit formula for the error terms, with the expectation of obtaining substantial cancellations when summing them over $q$. We begin with

$$
\sum_{\substack{M_{1}<m \leqslant M_{2} \\ m=a p n_{1}(\bmod q)}} 1=\frac{M_{2}-M_{1}}{q}+\psi\left(\frac{M_{1}-a \overline{p n}_{1}}{q}\right)-\psi\left(\frac{M_{2}-a \overline{p n}_{1}}{q}\right),
$$

where $\psi(\theta)=\theta-[\theta]-\frac{1}{2}$. To arrive at $U_{p}(M, N)$ we replace $\left(M_{2}-M_{1}\right) q^{-1}$, with the help of Lemma 3, by

$$
\frac{M_{2}-M_{1}}{q}=\frac{1}{\phi(q)} \sum_{\substack{M_{1}<m \leqslant M_{2} \\(m, q)=1}} 1+O\left(\frac{\tau(q)}{\phi(q)}\right) .
$$

The first term above contributes to $V_{p}(M, Q)$ exactly $U_{p}(M, Q)$, while the error term 
$O(\tau(q) / \phi(q))$ contributes

$$
\ll \sum_{Q<q \leqslant 2 Q} N^{2} \frac{\tau(q)}{\phi^{2}(q)} \ll N^{2} Q^{-1} \log Q
$$

Therefore we may write

$$
V_{p}(M, Q)=U_{p}(M, Q)+\widetilde{V}_{p}\left(M_{1}, Q\right)-\widetilde{V}_{p}\left(M_{2}, Q\right)+O\left(N^{2} Q^{-1} \log Q\right),
$$

where for $L=M_{1}$ or $M_{2}$ we define

$$
\tilde{V}_{p}(L, Q)=\sum_{\substack{Q<q \leqslant 2 Q \\(q, a p)=1}} \frac{1}{\phi(q)} \sum_{\substack{N / 2<n_{1}, n_{2}<2 N \\\left(n_{1} n_{2}, p q\right)=1 \\ 1 / 2<n_{1} / n_{2}<2}} f_{p}\left(n_{1}\right) f_{p}\left(n_{2}\right) \psi\left(\frac{L-a \overline{p n}_{1}}{q}\right)
$$

By Lemma 4 we approximate $\psi(\xi)$ by $A(\xi)$ with error $B(\xi)$ giving

$$
\tilde{V}_{p}(L, Q) \ll \Delta N^{2}+\sum_{h \neq 0} C_{h} \sum_{N / 2<n_{1}, n_{2}<2 N}\left|\sum_{\substack{Q<q \leqslant 2 Q \\\left(q, a p n_{1} n_{2}\right)=1}} \frac{1}{\phi(q)} e\left(h \frac{L-a \overline{p n}_{1}}{q}\right)\right| .
$$

We use $v \mid q^{\infty}$ to mean that each prime that divides $v$ also divides $q$. Since $q / \phi(q)=\sum_{v \mid q^{\infty}} y^{-1}$, we obtain, by Lemmas 5 and 6 and by partial summation,

$$
\begin{aligned}
& \sum_{\substack{Q<q \leqslant 2 Q \\
\left(q, a p n_{1} n_{2}\right)=1}} \frac{1}{\phi(q)} e\left(h \frac{L-a \overline{p n}_{1}}{q}\right)=\sum_{\substack{Q<q \leqslant 2 Q \\
\left(q, a p n_{1} n_{2}\right)=1}} \frac{1}{\phi(q)} e\left(h \frac{L p n_{1}-a}{q p n_{1}}\right) e\left(a h \frac{\bar{q}}{p n_{1}}\right) \\
& \ll Q^{-1}\left(1+\frac{|h| x}{p Q N}\right) \sup _{Q<Q_{1} \leqslant 2 Q}\left|\sum_{\substack{Q<q \leqslant Q_{1} \\
\left(q, a p n_{1} n_{2}\right)=1}} \frac{q}{\phi(q)} l\left(a h \frac{\bar{q}}{p n_{1}}\right)\right| \\
& \ll Q^{-1}\left(1+\frac{|h| x}{p Q N}\right) \sup _{Q<Q_{1} \leqslant 2 Q} \sum_{\substack{v \mid P(2 Q)^{x} \\
\left(v, a p n_{1} n_{2}\right)=1}} v^{-1}\left|\sum_{\substack{v-1 \\
\left(r, a p n_{1} n_{2}\right)^{-1}=1}} l\left(a h \frac{\overline{v r}}{p n_{1}}\right)\right| \\
& \ll Q^{-1}\left(1+\frac{|h| x}{p Q N}\right)\left(a h, p n_{1}\right)^{1 / 2}(p N)^{1 / 2} x^{\varepsilon} .
\end{aligned}
$$

Now, summing over $n_{1}, n_{2}<2 N$ and $h \neq 0$ with weight $C_{h}$, (14) with $\Delta=M Q^{-1} x^{-2 \varepsilon}$ yields

$$
\begin{aligned}
\tilde{V}_{p}(L, Q) & \ll \Delta N^{2}+Q^{-1}\left(1+\frac{x}{\Delta p Q N}\right)(a, p)^{1 / 2}(p N)^{1 / 2} N^{2} x^{2 \varepsilon} \\
& \ll M N^{2} Q^{-1} x^{-2 \varepsilon}+(a, p)^{1 / 2} p^{1 / 2} N^{5 / 2} Q^{-1} x^{5 \varepsilon}
\end{aligned}
$$


Hence relation (13) becomes

$$
\begin{aligned}
V_{p}(M, Q)=U_{p}(M, Q) & \\
& +O\left(N^{2} Q^{-1} x^{\varepsilon}+M N^{2} Q^{-1} x^{-2 \varepsilon}+(a, p)^{1 / 2} p^{1 / 2} N^{5 / 2} Q^{-1} x^{5 \varepsilon}\right) .
\end{aligned}
$$

$\S 10$. Rearrangement of $W_{p}(M, Q)$. By definition,

$$
W_{p}(M, Q)=\sum_{\substack{Q<q \leqslant 2 Q \\(q, a p)=1}} \sum_{\substack{M<m \leqslant 2 M \\(m, q)=1}} \sum_{\substack{\left(n_{1} n_{2}, p\right)=1 \\ y / p m<n_{1}, n_{2} \leqslant 2 y / p m \\ p m n_{1} \equiv p_{1}=n_{2} \equiv a(\bmod q)}} f_{p}\left(n_{1}\right) f_{p}\left(n_{2}\right) .
$$

Here, if $\left(n_{1}, n_{2}\right)=d>1$ then $d \geqslant p$. Therefore, such terms contribute at most

$$
O\left(M N^{2} Q^{-1} p^{-1}+M N \log x\right) .
$$

Now, let us consider $W_{p}^{*}(M, Q)$-the contribution to $W_{p}(M, Q)$ of terms with $\left(n_{1}, n_{2}\right)=1$. Notice that the range for $n_{1}, n_{2}$ is equal to

$$
\begin{aligned}
\mathscr{E}(q)=\left\{\left(n_{1}, n_{2}\right) ;\left(n_{1} n_{2}, p q\right)=1,\left(n_{1}, n_{2}\right)=1,\right. \\
\left.n_{1} \equiv n_{2}(\bmod q), \frac{N}{2}<n_{1}, n_{2}<2 N, \frac{1}{2}<\frac{n_{1}}{n_{2}}<2\right\},
\end{aligned}
$$

and for given $q, n_{1}, n_{2}$ the number of $m$ 's is given by (12) with the same notation for $M_{1}$ and $M_{2}$. We treat the main term $\left(M_{2}-M_{1}\right) / q$ as in Section 9 . On replacing it by

$$
\frac{1}{\phi(q)} \sum_{\substack{M \\(m, q)=1}} 1
$$

we make the total error $\ll N^{2} Q^{-1} \log Q$. Another error of order (16) is made when relaxing the condition $\left(n_{1}, n_{2}\right)=1$ in $\mathscr{E}(q)$. The latter operation is necessary to obtain

$$
T_{p}(M, Q)=\sum_{\substack{Q<q \leqslant 2 Q \\(q, a p)=1}} \frac{1}{\phi(q)} \sum_{\substack{(\bmod q) \\(l, q)=1}} \sum_{\substack{M<m \leqslant 2 M \\(m, q)=1}}\left(\sum_{\substack{y<p m n \leqslant 2 y \\ p m n \\=(1, \bmod q) \\(n, p)=1}} f_{p}(n)\right)^{2},
$$

which we consider as a main term for $W_{p}(M, Q)$. It is clear, by the above discussion, that we obtain

$$
\begin{aligned}
W_{p}(M, Q) & =T_{p}(M, Q)+2 \tilde{W}_{p}\left(M_{1}, Q\right)-2 \tilde{W}_{p}\left(M_{2}, Q\right) \\
& +O\left(M N^{2} Q^{-1} p^{-1}+N^{2} Q^{-1} \log Q+M N \log x\right),
\end{aligned}
$$


where for $L=M_{1}$ or $M_{2}$ we define

$$
\tilde{W}_{p}(L, Q)=\sum_{\substack{Q<q \leqslant 2 Q \\(q, a p)=1}} \sum_{\substack{\left(n_{1}, n_{2}\right) \varepsilon \mathcal{E}(q) \\ n_{1}>n_{2}}} f_{p}\left(n_{1}\right) f_{p}\left(n_{2}\right) \psi\left(\frac{L-a \bar{p} \bar{n}_{2}}{q}\right) .
$$

$\S 11$. Rearrangement of $\tilde{W}_{p}(L, Q)$. Now approximate $\psi(\xi)$ by $A(\xi)$, with error $B(\xi)$, and expand $A(\xi)$ and $B(\xi)$ into Fourier series (see Lemma 4) giving

$$
\tilde{W}_{p}(L, Q) \ll \Delta N^{2} \log N+\sum_{h=1}^{\infty} C_{h}\left|W_{p, h}(L, Q)\right|,
$$

where

$$
W_{p, h}(L, Q)=\sum_{\substack{Q<q \leqslant 2 Q \\(q, a p)=1}} \sum_{\substack{\left(n_{1}, n_{2}\right) \in \mathscr{E}(q) \\ n_{1}>n_{2}}} f_{p}\left(n_{1}\right) f_{p}\left(n_{2}\right) e\left(h \frac{L-a \overline{p n}}{q}\right) .
$$

Replace the condition $(q, p)=1$ by splitting up the summation over $q$ into $p-1$ arithmetic progressions $\alpha(\bmod p), 1 \leqslant \alpha<p$, and detect $(q, a)=1$ by the relation

$$
\sum_{\substack{\left.\left.v\right|_{v} ^{a} \\ v\right|_{q}}} \mu(v)= \begin{cases}1, & \text { if }(q, a)=1, \\ 0, & \text { if }(q, a)>1,\end{cases}
$$

to obtain

$$
W_{p, h}(L, Q)=\sum_{1 \leqslant \alpha<\rho} \sum_{v \mid a} \mu(v) W_{p, h}^{\alpha, v}(L, Q)
$$

with

$$
W_{p, h}^{\alpha, v}(L, Q)=\sum_{\substack{Q<q \leqslant 2 Q Q \\ q \equiv \alpha(\bmod p) \\ q \equiv 0\left(n_{1}, n_{2}\right) \in E(q) \\ n_{1}>n_{2}}} f_{p}\left(n_{1}\right) f_{p}\left(n_{2}\right) e\left(h \frac{L-a \overline{p n}_{2}}{q}\right)
$$

For $\left(n_{1}, n_{2}\right) \in \mathscr{E}(q)$, we reinterpret the condition $n_{1} \equiv n_{2}(\bmod q)$, by writing $n_{1}-n_{2}=q r$, so that $1 \leqslant r \leqslant N Q^{-1}, Q r<n_{1}-n_{2} \leqslant 2 Q r$,

$$
n_{1}-n_{2} \equiv \alpha r(\bmod p r), n_{1}-\mathrm{n}_{2} \equiv 0(\bmod v r) \quad \text { and }\left(n_{1} n_{2}, p r\right)=1 \text {. }
$$

We may therefore write

$$
W_{p, h}^{\alpha, v}(L, Q)=\sum_{1 \leqslant r \leqslant N / Q} \sum_{\left(n_{1}, n_{2}\right) \in \mathscr{F}(r)} f_{p}\left(n_{1}\right) f_{p}\left(n_{2}\right) e\left(h \frac{L-a \overline{p n}_{2}}{q}\right),
$$


where $q=\left(n_{1}-n_{2}\right) / r$ and the range of summation in the inner sum is

$$
\begin{gathered}
\mathscr{F}(r)=\left\{\left(n_{1}, n_{2}\right) ; \frac{N}{2}<n_{1}, n_{2}<2 N, n_{2}<n_{1}<2 n_{2},\right. \\
Q r<n_{1}-n_{2} \leqslant 2 Q r,\left(n_{1}, n_{2}\right)=1, \\
\left(n_{1} n_{2}, p r v\right)=1, n_{1}-n_{2} \equiv \alpha r(\bmod p r), \\
\left.n_{1} \equiv n_{2}(\bmod v r)\right\} .
\end{gathered}
$$

The variables $n_{2}$ and $n_{1}$ are of the same order of magnitude. Our intention is to spoil this 'symmetry' by an appeal to the combinatorial sieve identity. We apply Lemma 1 , with some parameter $G>p$ in place of $D$, giving

$$
\begin{aligned}
& \sum_{\left(n_{1}, n_{2}\right) \in \mathscr{F}(r)} f_{p}\left(n_{1}\right) f_{p}\left(n_{2}\right) e\left(h \frac{L-a \bar{p} n_{2}}{q}\right) \\
& \ll \sum_{g \leqslant G} \sum_{\left(n_{2}, p r v\right)=1}\left|\sum_{\substack{n_{1} \equiv 0(\bmod g) \\
\left(m_{1}, n_{2}\right) \in \mathscr{F}(r)}} e\left(h \frac{L-a \overline{p n_{2}}}{q}\right)\right| \\
& +\sum_{G / p \leqslant g \leqslant G} \sum_{\left(n_{2}, p r v\right)=1}\left|\sum_{\substack{n_{1} \equiv 0(\bmod g) \\
\left(n_{1}, n_{2}\right) \in \mathscr{\mathscr { F }}(r)}} f_{p(g)}\left(n_{1}\right) e\left(h \frac{L-a \overline{p n_{1}}}{q}\right)\right| \\
& =\sum_{g \leqslant G} \sum_{n_{2}}\left|\sum_{n_{1}}\right|+\sum_{G / p \leqslant g \leqslant G} \sum_{n_{2}}\left|\sum_{n_{1}} f\right|,
\end{aligned}
$$

say.

obtain, by Lemma 6 ,

§12. Estimate of $\sum_{g \leqslant G} \sum_{n_{2}}\left|\sum_{n_{1}}\right|$. Since $q \equiv \alpha(\bmod p)$ and $q \equiv \bar{r} n_{1}\left(\bmod n_{2}\right)$ we

$$
\frac{L-a \bar{p} n_{2}}{q} \equiv \frac{L p n_{2}-a}{q p n_{2}}+a \frac{\bar{q}}{p n_{2}} \equiv \frac{r\left(L p n_{2}-a\right)}{\left(n_{1}-n_{2}\right) p n_{2}}+a \frac{\left(r+\bar{\alpha} n_{2}\right) \bar{n}_{1}}{p n_{2}}(\bmod 1)
$$

Insert this into the inner sum $\sum_{n_{1}}$ and remove the factor $e\left(h r\left(L p n_{2}-a\right) / p n_{2}\left(n_{1}-n_{2}\right)\right)$ 
by partial summation, to obtain

$$
\begin{aligned}
\sum_{n_{1}} & =\sum_{\substack{n_{1} \equiv 0(\bmod g) \\
\left(n_{1}, n_{2}\right) \in \mathscr{F}(r)}} e\left(h r \frac{\left.L p n_{2}-a\right)}{p n_{2}\left(n_{1}-n_{2}\right)}\right) e\left(a h \frac{\left(r+\bar{\alpha} n_{2}\right) \overline{n_{1}}}{p n_{2}}\right) \\
& \ll\left(1+\frac{h x}{p N Q}\right) \sup _{\substack { N / 2<N_{1}<2 N \\
\begin{subarray}{c}{n_{1} \\
\left(n_{1}, n_{2}\right) \in \mathscr{F}(r) \leq N_{1}{ N / 2 < N _ { 1 } < 2 N \\
\begin{subarray} { c } { n _ { 1 } \\
( n _ { 1 } , n _ { 2 } ) \in \mathscr { F } ( r ) \leq N _ { 1 } } }\end{subarray}} e\left(a h \frac{\left(r+\bar{\alpha} n_{2}\right) \bar{n}_{1}}{p n_{2}}\right) \mid \\
& \ll\left(1+\frac{h x}{p N Q}\right)\left(a h, p n_{2}\right)^{1 / 2}(p N)^{1 / 2} x^{\varepsilon},
\end{aligned}
$$

by Lemma 5 . This yields

$$
\sum_{g \leqslant G} \sum_{n_{2}}\left|\sum_{n_{1}}\right| \ll\left(1+\frac{h x}{p N Q}\right)(a h, p)^{1 / 2} p^{1 / 2} \tau(h) G N^{3 / 2} x^{2 \varepsilon}
$$

§13. Estimate of $\sum_{G / P} \sum_{n_{2}}\left|\sum_{n_{1}} f\right|$. Since $q \equiv \alpha(\bmod p)$ and $q \equiv-\bar{r} n_{2}$ $\left(\bmod n_{1}\right)$, Lemma 6 yields

$$
\frac{L-a \overline{p n}_{1}}{q} \equiv \frac{L p n_{1}-a}{q p n_{1}}+a \frac{\bar{q}}{p n_{1}} \equiv \frac{\left(L p n_{1}-a\right) r}{p n_{1}\left(n_{1}-n_{2}\right)}+a \frac{\alpha \bar{n}_{1}}{p}-a r \frac{\overline{n_{2} p}}{n_{1}}(\bmod 1)
$$

Insert this into the inner sum $\sum_{n_{1}} f$ and remove the factor $e\left(h r\left(L p n_{1}-a\right) / p n_{1}\left(n_{1}-n_{2}\right)\right)$ by partial summation, to obtain

$$
\sum_{n_{2}}\left|\sum_{n_{1}} f\right| \ll\left(1+\frac{h x}{p N Q}\right) \sup _{N / 2<N_{1}<2 N} \sum_{\substack{N / 2<n_{2}<N \\\left(n_{2}, p r v\right)=1}}\left|\sum_{\substack{\left.n_{1} \\ \text { n. } \\\left(n_{1}, n_{2}\right) \in N_{1}\right) \mathscr{Y}(r)}} c\left(n_{1}\right) e\left(-a h r \frac{\overline{n_{2} p}}{\overline{n_{1}}}\right)\right|,
$$

where $c\left(n_{1}\right)=f_{p(g)}\left(n_{1}\right) e\left(a h \frac{\alpha \bar{n}_{1}}{p}\right)$ is independent of $n_{2}$. By the Cauchy-Schwarz inequality,

$$
\begin{aligned}
& \sum_{\substack{N / 2<n_{2}<N \\
\left(n_{2}, p r v\right)=1}}\left|\sum_{\substack{n_{n}=1 \\
\left(n_{1} \leq 0\left(N_{1} \\
\left(n_{1}, n_{2}\right) \in \bar{m}(r)\right.\right.}} c\left(n_{1}\right) e\left(-a h r \frac{\overline{n_{2} p}}{n_{1}}\right)\right| \\
& \left.\leqslant(2 N)^{1 / 2}\left\{\sum_{\substack{n^{\prime}, n^{\prime \prime} \leqslant N_{1} \\
n^{\prime}=0\left(n^{\prime \prime}=0(\bmod g) \\
\left(n^{\prime} n^{\prime \prime}, p\right)=1\right.}} \mid \sum_{\substack{\left(n^{\prime}, n\right) \in \operatorname{mig}(r) \\
\left(n^{\prime \prime}, n\right) \in \mathscr{F}(r)}} e\left(\operatorname{ahr}\left(\frac{\overline{n p}}{n^{\prime}}-\frac{\overline{n p}}{n^{\prime \prime}}\right)\right)\right)\right\}^{1 / 2}
\end{aligned}
$$

However,

$$
\frac{\overline{n p}}{n^{\prime}}-\frac{\overline{n p}}{n^{\prime \prime}} \equiv \frac{n^{\prime \prime}-n^{\prime}}{\left(n^{\prime}, n^{\prime \prime}\right)} \frac{\overline{n p}}{\left[n^{\prime}, n^{\prime \prime}\right]}(\bmod 1)
$$


Hence, by Lemma 5 ,

$$
\sum_{\substack{\left.n^{\prime}, n\right) \in \mathscr{F}(r) \\\left(n^{\prime \prime}, n\right) \in \mathscr{F}(r)}} e\left(a h r\left(\frac{\overline{n p}}{n^{\prime}}-\frac{\overline{n p}}{n^{\prime \prime}}\right)\right) \ll\left(a h\left(n^{\prime \prime}-n^{\prime}\right), n^{\prime} n^{\prime \prime}\right)^{1 / 2}\left(n^{\prime}, n^{\prime \prime}\right)^{-1} N x^{\varepsilon}
$$

Summation over $n^{\prime}$ and $n^{\prime \prime}$ yields

$$
\begin{aligned}
& \sum_{\substack{n^{\prime}, n^{\prime \prime}<2 N \\
n^{\prime \prime} \equiv n^{\prime \prime} \equiv 0(\bmod g)}}\left(a h\left(n^{\prime \prime}-n^{\prime}\right), n^{\prime} n^{\prime \prime}\right)^{1 / 2}\left(n^{\prime}, n^{\prime \prime}\right)^{-1} \\
& \ll g^{-1} N+g^{-1 / 2} \sum_{l_{1}<l_{2}<2 N / g}\left(a h\left(l_{2}-l_{1}\right), g l_{1} l_{2}\right)^{1 / 2} \\
& \ll g^{-1} N+(a h, g) g^{-5 / 2} N^{2} x^{\varepsilon}
\end{aligned}
$$

Gathering all the above results together we finally obtain

$$
\sum_{G / p} \sum_{i=g}\left|\sum_{n_{2}} f\right| \ll\left(G^{1 / 2} N^{3 / 2}+p^{1 / 4} G^{-1 / 4} N^{2} \tau(h)\right)\left(1+\frac{h x}{p N Q}\right) x^{\varepsilon} .
$$

§14. Estimate of $\tilde{W}_{p}(L, Q)$. Collecting (17), (18), (19), (20), (21) and (22) we obtain

$$
\begin{aligned}
\tilde{W}_{p}(L, Q) & \ll \Delta N^{2} \log N+\left(1+\frac{x}{p Q N \Delta}\right) \frac{p N}{Q}\left((a, p)^{1 / 2} p^{1 / 2} G N^{3 / 2}+p^{1 / 4} G^{-1 / 4} N^{2}\right) x^{3 \varepsilon} \\
& \ll M N^{2} Q^{-1} x^{-2 \varepsilon}+(a, p)^{1 / 10} p^{13 / 10} N^{29 / 10} Q^{-1} x^{7 \varepsilon}
\end{aligned}
$$

for $\Delta=M Q^{-1} x^{-3 \varepsilon}$ and $G=(a, p)^{-2 / 5} p^{-1 / 5} N^{2 / 5}$.

\$15. Estimate of the dispersion $D_{p}(M, Q)$. If we introduce results (15) and (23) into the definition of $D_{p}(M, Q)$ we obtain

$$
D_{p}(M, Q)=X_{p}(M, Q)+R_{p}(M, Q),
$$

where $X_{p}(M, Q)$ stands for the sum of the main terms, i.e.

$$
\begin{aligned}
X_{p}(M, Q) & =T_{p}(M, Q)-2 U_{p}(M, Q)+U_{p}(M, Q) \\
& =\sum_{\substack{Q<q \leqslant 2 Q \\
(q, a p)=1}} \sum_{\substack{M<m \leqslant 2 M \\
(m, q)=1}} \frac{1}{\phi(q)} \sum_{\substack{(\bmod q) \\
(l, q)=1}}\left(\sum_{\substack{y<p m n \leqslant 2 y \\
p m n=l(\bmod q) \\
(n, p)=1}} f_{p}(n)-\frac{1}{\phi(q)} \sum_{\substack{y<m n \leqslant 2 y \\
(n, p q)=1}} f_{p}(n)\right)^{2},
\end{aligned}
$$

and the error term $R_{p}(M, Q)$ is

$$
\begin{aligned}
R_{p}(M, Q) \ll M N^{2} Q^{-1} x^{-2 \varepsilon}+N^{2} Q^{-1} x^{\varepsilon} & +M N^{2} Q^{-1} p^{-1} \\
& +M N x^{\varepsilon}+Q x^{\varepsilon}+(a, p)^{1 / 10} p^{13 / 10} N^{29 / 10} Q^{-1} x^{7 \varepsilon}
\end{aligned}
$$


It remains to estimate $X_{p}(M, Q)$. For this, we appeal to the large sieve inequality (2). We first write

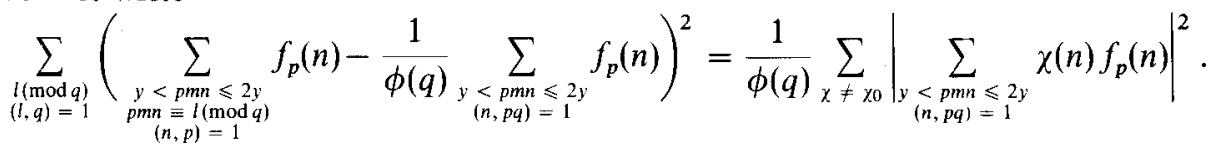

Summing this over $q$ and replacing each $\chi(\bmod q)$ by its induced primitive character $\chi^{*}(\bmod d), d \mid q$, we obtain

$$
\begin{aligned}
& \sum_{Q<q \leqslant 2 Q} \frac{1}{\phi^{2}(q)} \sum_{\chi \neq \chi 0}\left|\sum_{\substack{y, p m n \leqslant 2 y \\
(n, p q)=1}} \chi(n) f_{p}(n)\right|^{2} \\
& \ll Q^{-2}(\log Q)^{2} \sum_{e<2 Q} \sum_{1<d<2 Q / e} \sum_{\chi(\bmod d)}^{*}\left|\sum_{\substack{y<m n \leqslant 2 y \\
(n, e p)=1}} \chi(n) f_{p}(n)\right|^{2} .
\end{aligned}
$$

If $Q / e \leqslant(\log x)^{2 A+11}=H$, say, apply the Corollary to Lemma 7 , and if $Q / e>H$ apply the large sieve inequality (2), to show that this expression is

$$
\ll N^{2} Q^{-1} H \exp \left(-\frac{1}{2}(\log x)^{1 / 6}\right)+\left(1+\frac{N}{Q H}\right) N(\log x)^{2} ;
$$

whence

$$
X_{p}(M, Q) \ll M N^{2} Q^{-1}(\log x)^{-2 A-8}+M N(\log x)^{2},
$$

and finally

$$
\begin{aligned}
& D_{p}(M, Q) \ll M N^{2} Q^{-1}(\log x)^{-2 A-8}+N^{2} Q^{-1} x^{\varepsilon} \\
& +M N x^{\varepsilon}+Q x^{\varepsilon}+(a, p)^{1 / 10} p^{13 / 10} N^{29 / 10} Q^{-1} x^{7 \varepsilon} .
\end{aligned}
$$

§16. Conclusion. By (11),

$$
\begin{aligned}
\sum_{z_{0} \leqslant p<z} S_{p}(y, D, Q) & \leqslant \sum_{z 0 \leqslant p<z} \sum_{M} E_{p}(y, M, Q)+O(y \exp (-\sqrt{\log x})) \\
& \ll \sum_{z_{0} \leqslant p<z} \sum_{M}\left(Q M D_{p}(M, Q)\right)^{1 / 2}+x \exp (-\sqrt{\log x}) \\
& \ll \sum_{z o \leqslant p<z} \sum_{M}\left\{M N(\log x)^{-A-4}+M^{1 / 2} N x^{\varepsilon}+M N^{1 / 2} Q^{1 / 2} x^{\varepsilon}+M^{1 / 2} Q x^{\varepsilon}\right. \\
& \left.+(a, p)^{1 / 20} p^{13 / 20} M^{1 / 2} N^{29 / 20} x^{4 \varepsilon}\right\}+x \exp (-\sqrt{\log x}) \\
\ll x(\log x)^{-A-2}+z D^{-1 / 2} x^{1+\varepsilon}+D^{1 / 2} Q^{1 / 2} x^{1 / 2+\varepsilon} & +z^{1 / 2} D^{1 / 2} Q x^{\varepsilon}+z^{21 / 10} D^{-19 / 20} x^{29 / 20+5 \varepsilon}
\end{aligned}
$$

$\ll x(\log x)^{-A-2}$, 
on taking $D=x^{10 / 21-3 \varepsilon}$ for $Q \leqslant x^{11 / 21}$ and $z \leqslant x^{1 / 883}$. Hence by (7), (8) and (10) we obtain (5). This completes the proof of the theorem.

Remark. The limit for the present method turns out to be $Q=x^{10 / 19-\eta}$ in which case $z \leqslant x^{\dot{\delta}}$, with $\delta=\delta(\eta)$ a very small positive constant.

\section{References}

1. E. Bombieri. "On the large sieve", Mathematika, 12 (1965), 201-225.

2. X. Ding and C. D. Pan. "A new mean value theorem", Sci. Sinica, Special Issue (II), (1979), 149-161.

3. J. Friedlander and H. Iwaniec. "Quadratic polynomials and quadratic forms", Acta Math., 141 (1978), $1-15$.

4. P. X. Gallagher, "Bombieri's mean value theorem", Mathematika, 15 (1968), 1-6.

5. H. Halberstam and H. -E. Richert. Sieve Methods, (Academic Press, London-New York, 1974).

6. C. Hooley. "On the number of divisors of quadratic polynomials", Acta Math., 110 (1963), 97-114.

7. C. Hooley. "On the greatest prime factor of a cubic polynomial", J. Reine angew. Math., 303/304 (1978), 21-50.

8. H. Iwaniec. "Rosser's sieve", Acta Arith., 36 (1978), 171-202.

9. H. D. Kloosterman. "On the representation of numbers in the form $a x^{2}+b y^{2}+c z^{2}+d t^{2}$ ", Acta Math., 49 (1926), 407-464.

10. H. L. Montgomery. Topics in Multiplicative Number Theory, Lecture Notes in Math. 227 (Berlin and New York, 1971).

11. Y. Motohashi. "An induction principle for the generalization of Bombieri's Prime Number Theorem", Proc. Japan Acad., 52 (1976), 273-275.

12. H. -E. Richert. Lectures on Sieve Methods, (Tata Inst. of Fund. Research, 1976).

13. R. C. Vaughan. "On the estimation of trigonometric sums over primes and related questions", Institut Mittag-Leffler Report No. 9 (1977).

14. A. I. Vinogradov, "The density hypothesis for Dirichlet's L-series" (Russian), Izv. Akad. Nauk SSSR, Ser. Math. 29 (1965), 903-934; Corrigendum: ibid. 30 (1966), 719-720.

15. D. Wolke. "Über die mittlere Verteilung der Werte zahlentheoretischen Funktionen auf Restklassen I, Math. Annal., 202 (1973), 1-25.

Dr. E. Fouvry,

U.E.R. de Mathématiques et d'Informatique,

Université de Bordeaux I,

351, Cours de la Libération,

33405 Talence,

France.

Dr. H. Iwaniec,

U.E.R. de Mathématiques et d'Informatique,

Université de Bordeaux I,

351, Cours de la Libération, 33405 Talence,

France.
10H15: NUMBER THEORY; Multiplicative theory; Distribution of primes and of integers with specified multiplicative properties. 